\title{
Cumulative inactivated vaccine exposure and allergy development among children: a birth cohort from Japan
}

Kiwako Yamamoto-Hanada ${ }^{1,2^{*}}$ (D), Kyongsun Pak ${ }^{3}$, Mayako Saito-Abe ${ }^{1,2}$, Limin Yang ${ }^{1,2}$, Miori Sato ${ }^{1,2}$, Hidetoshi Mezawa ${ }^{2}$, Hatoko Sasaki ${ }^{2}$, Minaho Nishizato ${ }^{2}$, Mizuho Konishi², Kazue Ishitsuka², Kenji Matsumoto ${ }^{2,4}$, Hirohisa Saito ${ }^{2,4}$, Yukihiro Ohya ${ }^{1,2}$ and Japan Environment and Children's Study (JECS) Group

\begin{abstract}
Background: Adjuvants used in inactivated vaccines often upregulate type 2 immunity, which is dominant in allergic diseases. We hypothesised that cumulative adjuvant exposure in infancy may influence the development of allergies later in life by changing the balance of type 1/type 2 immunity. We examined the relationship between immunisation with different vaccine types and later allergic disease development.

Methods: We obtained information regarding vaccinations and allergic diseases through questionnaires that were used in The Japan Environment and Children's Study (JECS), which is a nationwide, multicentre, prospective birth cohort study that included 103,099 pregnant women and their children. We examined potential associations between the initial vaccination before 6 months of age and symptoms related to allergies at 12 months of age.

Results: Our statistical analyses included 56,277 children. Physician-diagnosed asthma was associated with receiving three (aOR 1.395, 95\% Cl 1.028-1.893) or four to five different inactivated vaccines (aOR 1.544, 95\% Cl 1.149-2.075), compared with children who received only one inactivated vaccine. Similar results were found for two questionnaire-based symptoms, i.e. wheeze (aOR 1.238, 95\% Cl 1.094-1.401; three vaccines vs. a single vaccine) and eczema (aOR 1.144, 95\% Cl 1.007-1.299; four or five vaccines vs. a single vaccine).

Conclusions: Our results, which should be cautiously interpreted, suggest that the prevalence of asthma, wheeze and eczema among children at 12 months of age might be related to the amount of inactivated vaccine exposure before 6 months of age. Future work should assess if this association is due to cumulative adjuvant exposure. Despite this possible association, we strongly support the global vaccination strategy and recommend that immunisations continue.
\end{abstract}

Trial registration: UMIN000030786.

Keywords: Adjuvant, Asthma, Eczema, Wheeze, Inactivated vaccine

\footnotetext{
* Correspondence: yamamoto-k@ncchd.go.jp

${ }^{1}$ Allergy Center, National Center for Child Health and Development, Tokyo, Japan

${ }^{2}$ Medical Support Center for the Japan Environment and Children's Study,

National Research Institute for Child Health and Development, Tokyo, Japan

Full list of author information is available at the end of the article
}

C C The Author(s). 2020 Open Access This article is licensed under a Creative Commons Attribution 4.0 International License, which permits use, sharing, adaptation, distribution and reproduction in any medium or format, as long as you give appropriate credit to the original author(s) and the source, provide a link to the Creative Commons licence, and indicate if changes were made. The images or other third party material in this article are included in the article's Creative Commons licence, unless indicated otherwise in a credit line to the material. If material is not included in the article's Creative Commons licence and your intended use is not permitted by statutory regulation or exceeds the permitted use, you will need to obtain permission directly from the copyright holder. To view a copy of this licence, visit http://creativecommons.org/licenses/by/4.0/. The Creative Commons Public Domain Dedication waiver (http://creativecommons.org/publicdomain/zero/1.0/) applies to the data made available in this article, unless otherwise stated in a credit line to the data. 


\section{Introduction}

Universal childhood vaccination is very important and plays a role in saving the lives of children. Vaccines stimulate the immune system to protect an individual against future infections. There is clear evidence that immunisation by the administration of vaccines is effective for controlling and preventing various life-threatening infectious diseases. Unlike live vaccines, inactivated vaccines often require adjuvants to generate an immune response because the immunogenicity of the inactivated vaccines is generally lower and their effective period is shorter compared with live vaccines [1]. Aluminium is the most commonly used adjuvant in inactivated vaccines [2]. However, aluminium adjuvants are known to induce type 2 immune responses. Clinical trials have tested all adjuvants that are currently used in vaccines for safety and effectiveness, and these adjuvants have been used safely in clinical practice for many years [3]. In a mouse model, it is well-known that IgE production is enhanced by presenting antigens with aluminium adjuvants [4]. For basic science research, it is common to enhance allergic reactions in animal models using aluminium adjuvants; however, the activated pathways and effects stimulated by aluminium adjuvants in people may differ from these models because findings from animals do not always translate into humans.

The association of inactivated vaccines with allergic diseases remains controversial. In support of this idea, Kiraly et al. showed that delayed diphtheria, tetanus and acellular pertussis (DTaP) vaccination, defined as giving the first dose after 90 days of age $(1$ month later than usual), was associated with reduced eczema and use of eczema medication in Australia [5]. Schlaud et al. reported that children in Germany who completed TDPHiHeP immunisation (tetanus, diphtheria, poliomyelitis, Haemophilus influenza type b; Hib, hepatitis B and pertussis) at the end of the first year of life had a lower risk of subsequently developing hay fever [6]. In contrast, two different studies reported that routine immunisation against pertussis in infancy did not increase the risk of asthma or wheeze $[7,8]$.

Unlike inactivated vaccines whose adjuvants may promote type 2 immunity, the bacillus Calmette-Guérin (BCG) vaccine promotes the development of type 1 helper $\mathrm{T}$ (Th1) and IL-17-producing helper $\mathrm{T}$ (Th17) cells [9]. Although some studies have suggested that BCG vaccination is protective against the development of allergic diseases, a systematic review and metaanalysis concluded that BCG vaccination was not associated with development of eczema, rhinoconjunctivitis, allergic sensitization, or allergic diseases in general [10]. Notably, that review included only retrospective cohort studies, cross-sectional studies and a small RCT, and did not include any prospective cohort studies; therefore, the evidence level of the review does not seem very high. Further study is needed to confirm or exclude the presence of an association between BCG vaccination and allergic diseases.

Allergies, such as pollinosis, are a serious health concern in Japan. The prevalence of asthma and eczema among Japanese children is $13.3 \%$ and $24.5 \%$, respectively [11]. According to a nationwide birth cohort study, about $36 \%$ of pregnant women in Japan have a history of pollinosis and $55.6 \%$ are IgE-sensitised to Japanese cedar [12]. Given the relatively high prevalence of allergies in Japan, it is important to investigate potential triggers and preventions for the development of this condition.

We hypothesised that adjuvant exposure through early immunisation (before 6 months of age) could have a crucial influence on the development of allergic diseases. This study addressed three research questions (RQs). Our first RQ (RQ1) was whether babies who receive their first inoculations with inactivated vaccines, and are consequently exposed to adjuvant, later develop more allergic diseases than those first inoculated with live vaccines (no adjuvant required) (RQ1). Our second RQ (RQ2) was whether simultaneous inoculation with a greater number of inactivated vaccines (as a proxy for adjuvant exposure) leads to a proportionately greater risk of subsequently developing allergic diseases. Finally, our third RQ (RQ3) was whether BCG vaccination reduces the risk of allergic diseases. To answer these RQs, we examined the relationship between initial (i.e. the first time in life; before 6 months of age) vaccine type exposure and later allergic disease development in the general population of Japan.

\section{Methods \\ Study population}

Although it is not a clinical trial, the Japan Environment and Children's Study (JECS) is registered with the University Hospital Medical Information Network Clinical Trials Registry, number UMIN000030786. The JECS is a nationwide, multicentre, prospective birth cohort study conducted by the Ministry of Environment of Japan, and its protocol and major hypotheses have been published as a protocol paper [12-15]. A total of 103,099 pregnant women were recruited from the general population of Japan for the JECS at 15 research centres covering a wide geographical area of Japan, from the north of the country (Hokkaido) to the south (Okinawa), from April 2011 to March 2014. The pregnancies of the recruited women resulted in 104,065 newborns. The present study was conducted as a part of the JECS, and we performed statistical analyses using the released data sets (jecs-ag-20160424 and jecs-an-20180131). 


\section{Ethics statement}

The JECS protocol was reviewed and approved by the Ministry of the Environment's Institutional Review Board on Epidemiological Studies and by the ethics committees of all participating institutions. Written informed consent was obtained from all participants (i.e. pregnant women or other caregivers). The JECS was conducted in accordance with the Helsinki Declaration and other nationally valid regulations and guidelines.

\section{Questionnaires and medical records}

Written questionnaires were mailed to caregivers during pregnancy and when each child was 6 months and 12 months of age. Caregivers answered questions regarding the mothers and infants during pregnancy and at ages 6 and 12 months. In addition, we compiled birth and neonatal information, including the obstetric outcomes, from the medical records provided by each participant's institution.

\section{Outcomes, exposures and covariates}

The outcome definitions for this study are defined in the file S1. Prevalence of allergic diseases (asthma, atopic dermatitis, food allergy and any other allergies) was assessed based on a parent-reported doctor's diagnosis, obtained from participant questionnaires when the children were 12 months old. Wheeze and eczema symptoms were evaluated at age 12 months based on a questionnaire from the International Study of Asthma and Allergies in Childhood (ISAAC) [16]. Vaccination history was obtained from the questionnaires completed at age 6 months. Caregivers of the children checked the types of initial vaccination on the questionnaire. Diphtheriatetanus-pertussis vaccine (DPT) was defined as a single vaccine. The types of polio vaccination administered in Japan differed by the date $[17,18]$. Live polio vaccine was administered until August, 2012. Subsequently, inactivated polio virus vaccine became available in September, 2012 and DPT-inactivated polio vaccine (IPV) in November, 2012. Therefore, based on the birth date, we categorised polio vaccines into either live or inactivated vaccine. The DPT-IPV was counted as another inactivated vaccine type, i.e. counted as two types.

\section{Target populations for statistical analysis}

For RQ1, we included the subset of infants whose initial vaccination was with inactivated vaccine(s) only and those whose initial vaccination was with live vaccine(s) only. For RQ2, we included infants whose first vaccination was with inactivated vaccines only. For RQ3, we included infants whose initial vaccination was with BCG only and those whose initial vaccination was with rotavirus only.

\section{Statistical analyses}

After excluding multiple births and cases with missing variables, we performed statistical analyses of the remaining data (Figure S1). Based on clinical experience and a review of past reports, we assessed the following factors as possible confounders in the regression analyses: maternal age, primiparity, maternal allergy history, smoking during pregnancy, pet ownership during pregnancy, sex of the child, feeding at 6 months, nursery school attendance, maternal education level, annual family income and place of recruitment. We checked the collinearity of the preliminarily selected confounders and included all the available variables in the model without performing variable selection. For each outcome variable of allergic features and for the exposure variables in each $R Q$, we conducted logistic regression analyses in which all potential confounders were included. We evaluated the adjusted odds ratios (aORs) and odds ratios (ORs) as measures of association and calculated 95\% confidence intervals (CIs) and $p$ values. The adjusted $p$ values were calculated by the Holm method. For RQ2, we performed a multiplicity adjustment by Dunnett's method, with the number of inactivated vaccines set to 1 as a reference. For each model, we checked multicollinearity with the variance inflation factor, using a cut-off value of 5 . In addition, as sensitivity analyses for RQ1 $(n=56,277)$ and RQ3 $(n=8012)$, we estimated aORs by implementing a multivariate imputation (MI) using a chained equations (MICE) algorithm with 20 iterations and using the $\mathrm{R}$ package MICE, version 3.3.0 [19]. We did not perform imputation for RQ2 because we were able to confirm the same tendency as RQ1 based on the MI results. All available confounders were used in the imputation equations.

This was an exploratory study to estimate the magnitude of associations between allergy and vaccination; therefore, we did not define the significance level of $p$ values. We also performed Bayesian multivariable logistic regression using the $\mathrm{R}$ package rstan, version 2.17.3 [20], to estimate the posterior distribution of the aORs. We applied multiple statistical analyses, i.e., both Frequentist and Bayesian approaches, to evaluate the association measures from multiple perspectives. We set vague priors for all coefficients using a normal distribution with a mean of 0 and a variance of 10,000, and generated a posterior distribution sample by Markov chain Monte Carlo methods (No-U-Turn sampler) with 11,000 iterations, four chains, four thinning intervals and 1000 warm-up times. We assessed the posterior distribution of aORs with 25th, 50th, and 75th percentiles, 95\% credible intervals (CrIs) and the probabilities of aOR $>1.0$, > 1.5 and $>2.0$. All statistical analyses were performed using R statistical software, version 3.5.1 (The R Foundation, Vienna, Austria) [21]. 


\section{Results}

The prevalence of wheeze, eczema and physiciandiagnosed asthma at 1 year of age for the entire cohort of study participants was $19.9 \%, 18.4-18.7 \%$ and $2.5-$ $2.6 \%$, respectively (see Table $\mathrm{S} 1$ ). The prevalence of allergic features by each RQ is described in Tables S5, S6 and S7.

\section{RQ1: initial immunisation with inactivated vaccines only}

\section{vs. with live vaccines only}

To answer the question of whether or not initial inoculation with inactivated vaccines could lead to more frequent development of allergic diseases compared with initial inoculation using live vaccines, we compared subjects whose first vaccination was with inactivated vaccine(s) only to those whose first vaccination was with live vaccine(s) only. Regarding their initial vaccination, 8165 children received live vaccine(s) only and 48,112 children received inactivated vaccine(s) only (Table S2). The children who received inactivated vaccine(s) only did not show any associations with allergic features at 1 year of age compared with those who received live vaccine(s) only (Table 1). Based on the results of a Bayesian multivariable logistic regression, the posterior probabilities of aOR > 1.0 were 0.642 for food allergy, 0.488 for asthma and 0.603 for eczema (none of these are significant; Table 2). The results for complete case and MI case data did not differ.

\section{RQ2: Initial immunisation with multiple inactivated vaccines}

RQ2 aimed to examine whether there was a dosedependent relationship between receiving multiple inactivated vaccines simultaneously at the time of first vaccination and a greater risk of later development of allergic diseases. The percentages of infants who received only one type (including the triple vaccine DPT), two, three and four to five different types of inactivated vaccines were $21.2 \%, 64.0 \%, 7.7 \%$ and $7.2 \%$, respectively (Table S3). Physician-diagnosed asthma was associated with receiving three types of inactivated vaccine (aOR $1.395,95 \%$ CI 1.028-1.893) and four or five types of inactivated vaccine (aOR 1.544, 95\% CI 1.149-2.075) (Table 3). ISAAC-based wheeze symptom was associated with receiving three different inactivated vaccines at the initial immunisation (aOR 1.238, 95\% CI 1.094-1.401), and ISAAC-based eczema was associated with receiving four or five different inactivated vaccines at the initial immunisation (aOR 1.144, 95\% CI 1.007-1.299). The posterior probabilities of aORs were also increased for asthma, wheeze and eczema in Bayesian multivariable logistic regression analyses (Table 4). In particular, regarding the association of asthma with the administration of four or five types of inactivated vaccine at the initial immunisation, the posterior probability of aOR $>1.0$ was 1.000 , that of $\mathrm{aOR}>1.5$ was 0.581 and that of $\mathrm{aOR}>2.0$ was 0.012 (95\% CrI 1.198-1.967). As for eczema, the posterior probability of aOR $>1.0$ was $0.992(95 \% \mathrm{CrI}$ 1.027-1.269).

\section{RQ3: BCG vaccination vs. rotavirus vaccination}

RQ3 aimed to examine whether BCG vaccination as the initial immunisation reduces the risk of subsequent allergic diseases. The statistical analyses for RQ3 included 6093 infants vaccinated with BCG only and 1919 infants immunised with rotavirus vaccine only at their initial immunisations (Table S4). There were no differences in the allergic features between children whose initial vaccination was with BCG only and those whose initial immunisation was with rotavirus vaccine only, in multivariable logistic regression analyses of both complete case data and multiple imputation (MI) data (Table 5). In the analyses using Bayesian multivariable logistic regression, the posterior probability of aOR $>1.0$ was 0.841 , that of $\mathrm{aOR}>1.5$ was 0.165 and that of $\mathrm{aOR}>2.0$ was

Table 1 Results of univariable and multivariable logistic regression for allergic features associated with initial inactivated vaccine compared with initial live vaccine only, in the JECS birth cohort (RQ1)

\begin{tabular}{|c|c|c|c|c|c|c|c|c|c|c|c|c|}
\hline \multirow{2}{*}{$\begin{array}{l}\text { Allergy features } \\
\text { at } 1 \text { year old }\end{array}$} & \multicolumn{4}{|c|}{ Univariable } & \multicolumn{4}{|c|}{ Multivariable for complete cases } & \multicolumn{4}{|c|}{ Multivariable for multiple imputed data } \\
\hline & $\mathrm{OR}$ & Lower 95\% Cl & Upper 95\% Cl & $p$ value & $\mathrm{aOR}$ & Lower $95 \% \mathrm{Cl}$ & Upper $95 \% \mathrm{Cl}$ & $p$ value & $\mathrm{aOR}$ & Lower 95\% Cl & Upper $95 \% \mathrm{Cl}$ & $p$ value \\
\hline Allergic disease & 0.984 & 0.935 & 1.035 & 0.521 & 0.994 & 0.940 & 1.051 & 0.832 & 0.985 & 0.935 & 1.037 & 0.553 \\
\hline $\begin{array}{l}\text { Atopic } \\
\text { dermatitis }\end{array}$ & 0.909 & 0.813 & 1.016 & 0.093 & 0.943 & 0.834 & 1.065 & 0.341 & 0.914 & 0.816 & 1.023 & 0.118 \\
\hline Food allergy & 1.008 & 0.947 & 1.074 & 0.805 & 1.013 & 0.946 & 1.084 & 0.720 & 1.011 & 0.948 & 1.078 & 0.736 \\
\hline Asthma & 1.027 & 0.885 & 1.192 & 0.724 & 0.989 & 0.840 & 1.164 & 0.894 & 1.033 & 0.887 & 1.203 & 0.679 \\
\hline Wheeze & 1.001 & 0.944 & 1.062 & 0.963 & 0.980 & 0.917 & 1.047 & 0.550 & 0.991 & 0.932 & 1.054 & 0.776 \\
\hline Eczema & 0.978 & 0.921 & 1.039 & 0.474 & 1.009 & 0.944 & 1.078 & 0.797 & 0.992 & 0.933 & 1.054 & 0.785 \\
\hline
\end{tabular}

$O R$ odds ratio, $a O R$ adjusted odds ratio, $\mathrm{Cl}$ confidence interval

aOR and $95 \%$ Cls were estimated with potential confounders including maternal age, primiparity, maternal allergy history, smoking during pregnancy, pet ownership during pregnancy, sex of child, feeding at 6 months, nursery school attendance, maternal education level, annual family income and place of recruitment 
Table 2 Results of Bayesian multivariable logistic regression for allergic symptoms using vaccine types and covariates for complete cases (RQ1)

\begin{tabular}{|c|c|c|c|c|c|c|c|c|}
\hline Outcome & aOR P25 & aOR P50 & aOR P75 & 95\% Crl lower & 95\% Crl upper & $\operatorname{Pr}(\mathrm{aOR}>1.0)$ & $\operatorname{Pr}(a O R>1.5)$ & $\operatorname{Pr}(\mathrm{aOR}>2.0$ \\
\hline Allergic disease & 0.976 & 0.995 & 1.013 & 0.941 & 1.051 & 0.420 & $<0.0001$ & $<0.0001$ \\
\hline Atopic dermatitis & 0.905 & 0.943 & 0.983 & 0.834 & 1.064 & 0.177 & $<0.0001$ & $<0.0001$ \\
\hline Food Allergy & 0.989 & 1.013 & 1.037 & 0.947 & 1.084 & 0.642 & $<0.0001$ & $<0.0001$ \\
\hline Asthma & 0.936 & 0.990 & 1.046 & 0.843 & 1.167 & 0.448 & $<0.0001$ & $<0.0001$ \\
\hline Wheeze & 0.958 & 0.980 & 1.003 & 0.917 & 1.047 & 0.275 & $<0.0001$ & $<0.0001$ \\
\hline Eczema & 0.986 & 1.009 & 1.032 & 0.947 & 1.078 & 0.603 & $<0.0001$ & $<0.0001$ \\
\hline
\end{tabular}

Parameters: iterations $=11000$, chains $=4$, thinning $=4$, warm-ups $=1000$

$a O R$ adjusted odds ratio, $C r l$ credible interval, $P r$ probability, $P 2525$ th percentile, $P 5050$ th percentile, $P 7575$ th percentile

aORs were estimated with potential confounders including maternal age, primiparity, maternal allergy history, smoking during pregnancy, pet ownership during pregnancy, sex of child, feeding at 6 months, nursery school attendance, maternal education level, annual family income and place of recruitment

0.008 (95\% CrI 0.809-1.832) for asthma (Table 6). The results for complete case and MI case data did not differ.

\section{Discussion}

\section{Main findings}

The results relating to RQ2 are the primary finding of this study. A logistic regression analysis showed that physician-diagnosed asthma, ISAAC-based wheeze and ISAAC-based eczema in 12-month-old children were each associated with an initial inoculation in children aged less than 6 months of multiple types of inactivated vaccine as compared with an initial inoculation of a single type of inactivated vaccine. By using another statistical analysis, i.e. Bayesian multivariable logistic regression, the posterior probabilities of $\mathrm{aOR}>1.0, \mathrm{aOR}>1.5$ and even aOR $>2.0$ confirmed the association of asthma with initial inoculations of four or five types of inactivated vaccines to be significantly high. It is possible that higher cumulative adjuvant exposures from initial immunisation with greater numbers of inactivated vaccines might influence an

Table 3 Results of univariable and multivariable logistic regression for allergic features associated with number of initial inactivated vaccines received, in the JECS birth cohort (RQ2)

\begin{tabular}{|c|c|c|c|c|c|c|c|c|c|}
\hline \multirow{2}{*}{$\begin{array}{l}\text { Allergy features } \\
\text { at } 1 \text { year old }\end{array}$} & \multirow{2}{*}{$\begin{array}{l}\text { Number of } \\
\text { inactivated } \\
\text { vaccines }\end{array}$} & \multicolumn{4}{|c|}{ Univariable } & \multicolumn{4}{|c|}{ Multivariable for complete cases } \\
\hline & & $\mathrm{OR}$ & Lower $95 \% \mathrm{Cl}$ & Upper 95\% Cl & $p$ value & $\mathrm{aOR}$ & Lower 95\% Cl & Upper 95\% Cl & $p$ value \\
\hline \multirow[t]{3}{*}{ Allergic disease } & 2 vs. 1 & 1.012 & 0.953 & 1.072 & 0.932 & 0.993 & 0.932 & 1.059 & 0.710 \\
\hline & 3 vs. 1 & 1.031 & 0.934 & 1.137 & 0.932 & 1.044 & 0.938 & 1.162 & 0.686 \\
\hline & 4 or 5 vs. 1 & 1.075 & 0.973 & 1.188 & 0.263 & 1.098 & 0.985 & 1.223 & 0.125 \\
\hline \multirow[t]{3}{*}{ Atopic dermatitis } & 2 vs. 1 & 0.957 & 0.837 & 1.093 & 0.683 & 0.947 & 0.820 & 1.094 & 1.000 \\
\hline & 3 vs. 1 & 1.116 & 0.900 & 1.384 & 0.683 & 1.076 & 0.851 & 1.360 & 1.000 \\
\hline & 4 or 5 vs. 1 & 1.113 & 0.893 & 1.386 & 0.683 & 1.095 & 0.866 & 1.385 & 1.000 \\
\hline \multirow[t]{3}{*}{ Food allergy } & 2 vs. 1 & 0.939 & 0.875 & 1.009 & 0.075 & 0.959 & 0.888 & 1.035 & 0.579 \\
\hline & 3 vs. 1 & 0.886 & 0.784 & 1.002 & 0.059 & 0.965 & 0.847 & 1.101 & 1.000 \\
\hline & 4 or 5 vs. 1 & 0.914 & 0.807 & 1.036 & 0.089 & 0.963 & 0.842 & 1.101 & 1.000 \\
\hline \multirow[t]{3}{*}{ Asthma } & 2 vs. 1 & 1.278 & 1.063 & 1.538 & 0.002 & 1.201 & 0.981 & 1.470 & 0.034 \\
\hline & 3 vs. 1 & 1.662 & 1.268 & 2.179 & $<0.0001$ & 1.395 & 1.028 & 1.893 & 0.021 \\
\hline & 4 or 5 vs. 1 & 1.800 & 1.375 & 2.356 & $<0.0001$ & 1.544 & 1.149 & 2.075 & 0.002 \\
\hline \multirow[t]{3}{*}{ Wheeze } & 2 vs. 1 & 1.035 & 0.966 & 1.109 & 0.240 & 0.990 & 0.916 & 1.069 & 0.752 \\
\hline & 3 vs. 1 & 1.286 & 1.152 & 1.435 & $<0.0001$ & 1.238 & 1.094 & 1.401 & $<0.0001$ \\
\hline & 4 or 5 vs. 1 & 1.248 & 1.114 & 1.397 & $<0.0001$ & 1.130 & 0.995 & 1.283 & 0.046 \\
\hline \multirow[t]{3}{*}{ Eczema } & 2 vs. 1 & 1.021 & 0.952 & 1.096 & 0.485 & 1.006 & 0.932 & 1.086 & 0.851 \\
\hline & 3 vs. 1 & 1.086 & 0.966 & 1.220 & 0.191 & 1.113 & 0.980 & 1.261 & 0.095 \\
\hline & 4 or 5 vs. 1 & 1.116 & 0.992 & 1.257 & 0.084 & 1.144 & 1.007 & 1.299 & 0.038 \\
\hline
\end{tabular}

$O R$ odds ratio, $a O R$ adjusted odds ratio, $C l$ confidence interval

aOR and $95 \% \mathrm{Cls}$ were estimated with potential confounders including maternal age, primiparity, maternal allergy history, smoking during pregnancy, pet ownership during pregnancy, sex of child, feeding at 6 months, nursery school attendance, maternal education level, annual family income and place of recruitment. The adjusted $p$ values were calculated by Holm method. Results of $p$ value $<0.05$ are shown in bold 
Table 4 Results of Bayesian multivariable logistic regression for allergic features associated with number of initial inactivated vaccines for complete cases (RQ2)

\begin{tabular}{|c|c|c|c|c|c|c|c|c|c|}
\hline & $\begin{array}{l}\text { Variable: number of } \\
\text { vaccines }\end{array}$ & $\begin{array}{l}\text { aOR } \\
\mathrm{P} 25\end{array}$ & $\begin{array}{l}\text { aOR_ } \\
\mathrm{P}_{5}\end{array}$ & $\begin{array}{l}\text { aOR } \\
\text { P75 }\end{array}$ & $\begin{array}{l}95 \% \mathrm{Crl}_{-} \\
\text {lower }\end{array}$ & $\begin{array}{l}95 \% \mathrm{Crl}_{-} \\
\text {upper }\end{array}$ & $\begin{array}{l}\operatorname{Pr}(\mathrm{aOR}> \\
1.0)\end{array}$ & $\begin{array}{l}\operatorname{Pr}(\mathrm{aOR}> \\
1.5)\end{array}$ & $\begin{array}{l}\operatorname{Pr}(a O R> \\
2.0)\end{array}$ \\
\hline \multirow[t]{3}{*}{ Allergic disease } & 2 vs. 1 & 0.975 & 0.993 & 1.011 & 0.941 & 1.047 & 0.397 & $<0.0001$ & $<0.0001$ \\
\hline & 3 vs. 1 & 1.012 & 1.045 & 1.077 & 0.956 & 1.141 & 0.825 & $<0.0001$ & $<0.0001$ \\
\hline & 4 or 5 vs. 1 & 1.063 & 1.097 & 1.133 & 1.003 & 1.201 & 0.978 & $<0.0001$ & $<0.0001$ \\
\hline \multirow{3}{*}{$\begin{array}{l}\text { Atopic } \\
\text { dermatitis }\end{array}$} & 2 vs. 1 & 0.909 & 0.947 & 0.989 & 0.841 & 1.067 & 0.194 & $<0.0001$ & $<0.0001$ \\
\hline & 3 vs. 1 & 1.002 & 1.073 & 1.147 & 0.885 & 1.300 & 0.758 & 0.001 & $<0.0001$ \\
\hline & 4 or 5 vs. 1 & 1.023 & 1.095 & 1.170 & 0.810 & 1.330 & 0.815 & 0.001 & $<0.0001$ \\
\hline \multirow[t]{3}{*}{ Food allergy } & 2 vs. 1 & 0.939 & 0.959 & 0.980 & 0.899 & 1.020 & 0.090 & $<0.0001$ & $<0.0001$ \\
\hline & 3 vs. 1 & 0.930 & 0.965 & 1.002 & 0.864 & 1.074 & 0.263 & $<0.0001$ & $<0.0001$ \\
\hline & 4 or 5 vs. 1 & 0.927 & 0.963 & 1.000 & 0.862 & 1.076 & 0.250 & $<0.0001$ & $<0.0001$ \\
\hline \multirow[t]{3}{*}{ Asthma } & 2 vs. 1 & 1.133 & 1.202 & 1.275 & 1.015 & 1.434 & 0.982 & 0.007 & $<0.0001$ \\
\hline & 3 vs. 1 & 1.279 & 1.392 & 1.518 & 1.072 & 1.799 & 0.992 & 0.281 & 0.004 \\
\hline & 4 or 5 vs. 1 & 1.413 & 1.538 & 1.676 & 1.198 & 1.967 & 1.000 & 0.581 & 0.019 \\
\hline \multirow[t]{3}{*}{ Wheeze } & 2 vs. 1 & 0.969 & 0.990 & 1.012 & 0.929 & 1.056 & 0.377 & $<0.0001$ & $<0.0001$ \\
\hline & 3 vs. 1 & 1.195 & 1.237 & 1.281 & 1.116 & 1.374 & 1.000 & $<0.0001$ & $<0.0001$ \\
\hline & 4 or 5 vs. 1 & 1.089 & 1.129 & 1.171 & 1.016 & 1.254 & 0.987 & $<0.0001$ & $<0.0001$ \\
\hline \multirow[t]{3}{*}{ Eczema } & 2 vs. 1 & 0.985 & 1.007 & 1.029 & 0.945 & 1.072 & 0.578 & $<0.0001$ & $<0.0001$ \\
\hline & 3 vs. 1 & 1.072 & 1.112 & 1.153 & 0.999 & 1.234 & 0.974 & $<0.0001$ & $<0.0001$ \\
\hline & 4 or 5 vs. 1 & 1.103 & 1.144 & 1.187 & 1.027 & 1.269 & 0.992 & $<0.0001$ & $<0.0001$ \\
\hline
\end{tabular}

Parameters: iterations $=11000$, chains $=4$, thinning $=4$, warm-ups $=1000$.

$a O R$ adjusted odds ratio, $\mathrm{Crl}$ credible interval, $\mathrm{Pr}$ probability, $P 25$ 25th percentile, $P 50$ 50th percentile, $P 75$ 75th percentile

Results of $95 \% \mathrm{Crl}>1.0$ and probability $>0.975$ are shown in bold

aORs were estimated with potential confounders including maternal age, primiparity, maternal allergy history, smoking during pregnancy, pet ownership during pregnancy, sex of child, feeding at 6 months, nursery school attendance, maternal education level, annual family income and place of recruitment

individual's immunological status and lead to the development of a later allergic response.

\section{Inactivated vaccines}

Related results have been reported by several other investigators. Kiraly et al. showed that delayed DTaP vaccination was associated with reduced eczema in Australia [5], and McDonald et al. found that delayed administration of the first dose of whole-cell DPT immunisation in childhood was negatively associated with the development of asthma [22]. In contrast, studies by Spycher et al. and by Vogt et al. each reported that routine immunisation against pertussis in infancy did not

Table 5 Results of univariable and multivariable logistic regression for allergic features associated with initial live polio vaccine only compared with initial BCG vaccine only, in the JECS birth cohort (RQ3)

\begin{tabular}{|c|c|c|c|c|c|c|c|c|c|c|c|c|}
\hline \multirow{2}{*}{$\begin{array}{l}\text { Allergy features } \\
\text { at } 1 \text { year old }\end{array}$} & \multicolumn{4}{|c|}{ Univariable } & \multicolumn{4}{|c|}{ Multivariable for complete cases } & \multicolumn{4}{|c|}{ Multivariable for multiple imputed data } \\
\hline & OR & $\begin{array}{l}\text { Lower } 95 \% \\
\mathrm{Cl}\end{array}$ & $\begin{array}{l}\text { Upper 95\% } \\
\mathrm{Cl}\end{array}$ & $\begin{array}{l}p \\
\text { value }\end{array}$ & $\mathrm{aOR}$ & $\begin{array}{l}\text { Lower } \\
95 \% \mathrm{Cl}\end{array}$ & $\begin{array}{l}\text { Upper 95\% } \\
\text { Cl }\end{array}$ & $\begin{array}{l}p \\
\text { value }\end{array}$ & $\mathrm{aOR}$ & $\begin{array}{l}\text { Lower 95\% } \\
\mathrm{Cl}\end{array}$ & $\begin{array}{l}\text { Upper } 95 \% \\
\text { Cl }\end{array}$ & $\begin{array}{l}p \\
\text { value }\end{array}$ \\
\hline Allergic disease & 1.032 & 0.924 & 1.153 & 0.578 & 0.970 & 0.851 & 1.105 & 0.648 & 0.990 & 0.123 & 7.966 & 0.868 \\
\hline $\begin{array}{l}\text { Atopic } \\
\text { dermatitis }\end{array}$ & 0.872 & 0.678 & 1.122 & 0.287 & 0.889 & 0.663 & 1.192 & 0.430 & 0.833 & 0.087 & 7.936 & 0.191 \\
\hline Food allergy & 1.150 & 1.005 & 1.315 & 0.042 & 1.060 & 0.906 & 1.242 & 0.466 & 1.089 & 0.132 & 9.015 & 0.259 \\
\hline Asthma & 0.770 & 0.543 & 1.092 & 0.142 & 1.234 & 0.822 & 1.852 & 0.311 & 1.148 & 0.106 & 12.486 & 0.484 \\
\hline Wheeze & 0.914 & 0.802 & 1.041 & 0.175 & 1.068 & 0.912 & 1.2525 & 0.413 & 1.089 & 0.132 & 9.009 & 0.253 \\
\hline Eczema & 0.987 & 0.865 & 1.127 & 0.845 & 0.887 & 0.759 & 1.037 & 0.131 & 0.930 & 0.113 & 7.674 & 0.327 \\
\hline
\end{tabular}

$O R$ odds ratio, $a O R$ adjusted odds ratio, $\mathrm{Cl}$ confidence interval

aOR and $95 \% \mathrm{Cls}$ were estimated with potential confounders including maternal age, primiparity, maternal allergy history, smoking during pregnancy, pet ownership during pregnancy, sex of child, feeding at 6 months, nursery school attendance, maternal education level, annual family income and place of recruitment 
Table 6 Results of Bayesian multivariable logistic regression for allergic features associated with initial rotavirus vaccine only compared with initial BCG only, in the JECS birth cohort for complete cases (RQ3)

\begin{tabular}{lllllllll}
\hline Outcome & aOR P25 & aOR P50 & aOR P75 & $95 \%$ Crl lower & $95 \%$ Crl upper & $\operatorname{Pr}(\mathrm{aOR}>1.0)$ & $\operatorname{Pr}(\mathrm{aOR}>1.5)$ & $\operatorname{Pr}(\mathrm{aOR}>2.0)$ \\
\hline Allergic disease & 0.928 & 0.971 & 1.015 & 0.851 & 1.105 & 0.327 & $<0.0001<$ & $<0.0001$ \\
Atopic dermatitis & 0.798 & 0.884 & 0.977 & 0.656 & 1.181 & 0.205 & $<0.0001<0.0001$ \\
Food allergy & 1.005 & 1.060 & 1.119 & 0.904 & 1.234 & 0.770 & $<0.0001<$ & $<0.0001$ \\
Asthma & 1.069 & 1.230 & 1.410 & 0.809 & 1.823 & 0.841 & 0.165 & 0.008 \\
Wheeze & 1.012 & 1.069 & 1.129 & 0.912 & 1.250 & 0.795 & $<0.0001<$ & $<0.0001$ \\
Eczema & 0.839 & 0.887 & 0.934 & 0.758 & 1.036 & 0.063 & $<0.0001<$ & $<0.0001<$ \\
\hline
\end{tabular}

Parameters: iterations $=11000$, chains $=4$, thinning $=4$, warm-ups $=1000$

$a O R$ adjusted odds ratio, $C r l$ credible interval, $P r$ probability, $P 2525$ th percentile, $P 5050$ th percentile, $P 7575$ th percentile

aORs were estimated with potential confounders including maternal age, primiparity, maternal allergy history, smoking during pregnancy, pet ownership during pregnancy, sex of child, feeding at 6 months, nursery school attendance, maternal education level, annual family income and place of recruitment

increase the risk of asthma or wheeze [7, 8]. Notably, these studies did not evaluate all inactivated vaccines but instead focused on only DPT, and their results were inconsistent. Here, we focused on initial vaccination with any type of inactivated vaccine during infancy to obtain the results presented above. Another report also examined the effect of multiple vaccinations on allergic diseases, but their results were different: Schlaud et al. reported that children with sufficient TDPHiHeP (tetanus, diphtheria, poliomyelitis, Hib, hepatitis B and pertussis) vaccination by the end of the first year of life had a lower risk of later developing hay fever [6]. Unlike the present work, that study included not only vaccines administered in the initial immunisation but also vaccines administered later during the first year of life. The discrepancy between their findings and our results may be due to the fact that the immune system develops and matures gradually over the course of infancy [23].

\section{More types of inactivated vaccines}

Because inactivated vaccines generally contain adjuvants, our finding that initial immunisation with more types of inactivated vaccines is associated with the development of asthma or eczema at 12 months of age may implicate adjuvant exposure as a potential risk factor for the development of allergic diseases. We speculate that the mechanism for this association is the induction of type 2 immunological responses by adjuvants. However, not many cases of physician-diagnosed asthma are caused by type 2 immunity, including IgE-mediated allergic reactions at 12 months of age [24]. The present results regarding the effect of vaccination on physician-diagnosed asthma may be due to non-specific inflammation that is independent of type 2 immunity. In support of this possibility, Kashiwagi et al. reported that DPT/Hib/PCV immunisation increased the serum levels of a proinflammatory cytokine, IL-1 $\beta$, compared with immunisation with DPT alone [25]. Although a weak association was found between eczema and initial immunisation with multiple inactive vaccines, further studies should be conducted to elucidate whether the effects of initial immunisation with multiple inactivated vaccines on asthma development persist after infancy, when most asthmatic children become type 2 immunity-dominant and IgE-dependent.

\section{BCG vaccination (live vaccine)}

We did not generate any significant results in our comparison of initial immunisation with live versus inactivated vaccines for RQ1, suggesting that $B C G$ vaccination may not have a preventive effect on the development of allergies. However, because BCG vaccination is known to promote Th1/Th17 immunity, we directly examined the effect of the initial immunisation being with BCG as RQ3 [9]. Our results failed to confirm the hypothesis that BCG vaccination of infants reduces the risk of development of allergic diseases. These findings agree with those of a systematic review and meta-analysis that concluded that BCG vaccine was not associated with the development of eczema, rhinoconjunctivitis, allergic sensitisation or allergies in general [10]. However, they differ from those of two other studies. Adults in Finland with a history of Mycobacterium tuberculosis (TB) infection prior to age 17 years had a lower prevalence of asthma than those without a prior TB infection [26]. In addition, Shirakawa et al. found that positive tuberculin responses were associated with a lower prevalence of asthma, a lower total IgE titre, lower Th2 cytokine (i.e. IL-4, IL-10 and IL-13) levels and a higher Th1 cytokine (IFN- $\gamma$ ) level in Japanese school children [27]. These two studies included adolescent and adult populations, whereas our study evaluated only young infants aged up to 1 year $[26,27]$. The inconsistent results might stem from the different timings of outcome assessment between studies.

\section{Adjuvants}

Adjuvants are essential to enhancing the adaptive immune response in the case of inactivated vaccines [28]. Aluminium is the most commonly used adjuvant in human inactivated vaccines because this adjuvant stimulates type 2 immune responses [2, 28]. Ohishi et al. 
found that the use of hydroxypropyl- $\beta$-cyclodextrin (HP$\beta-C D)$ as a vaccine adjuvant enhanced IgG antibody responses to a similar degree as the use of alum as an adjuvant but it induced fewer antigen-specific IgE responses compared with alum [29]. Although we were unable to identify the mechanism of how inactivated vaccines alter immunity because our study was epidemiological, we speculate that it involved the induction of type 2 responses. Additional studies are needed to confirm a causative link between adjuvants, type $2 \mathrm{im}$ mune responses and the development of allergic diseases. If this mechanism is confirmed, the development of alternate adjuvants that induce fewer type 2 responses might reduce the induction of allergy by initial immunisation with inactivated vaccines.

\section{Limitations}

This study has several limitations. First, we could not confirm the information provided by the vaccine manufacturers, so we could not calculate an accurate cumulative dose of adjuvant based on the vaccine type. Notably, the Hib vaccine used in Japan $\left(\mathrm{ActHIB}^{\circ}\right)$ does not include an adjuvant despite being an inactivated vaccine. However, because our RQs were based on distinguishing between live and inactivated vaccines, which we could do from the questionnaires, we were still able to complete our study, which focused on vaccination rather than adjuvant exposure. Second, the relatively large amount of missing data in our study resulted in a response bias. To address this limitation, we applied multiple imputation to account for missing values. We found no differences in the results between the complete case data and the multiple imputation data. Third, recall bias is a major concern in birth cohorts with selfreported questionnaires, which means that the prevalence of allergic diseases may have been underestimated. In addition, it was sometimes unclear at what ages in months the infants received their initial immunisation and developed allergic symptoms. The exact cause-andeffect relationship between exposures and outcomes was unclear. Notably, this study was unable to assess if children had allergic features during the first few months of life prior to vaccination. Because of this limitation, it is not possible to determine whether the reported allergies developed as a result of the effects of vaccination, so this study can evaluate only associations. Furthermore, the ISAAC questionnaire is not officially meant to be used for 1-year-old infants. However, various epidemiological studies have applied the ISAAC questionnaire for a wide range of ages [30]. Fourth, it was not possible to include children who had no clear history of immunisation in our comparisons. Fifth, we did not include all potential confounders, such as hospital visit frequency, in our analyses. The strength of this study is that it was conducted using a huge sample recruited from the general population across Japan in a nationwide prospective birth cohort study. Further studies are needed to validate our results.

\section{Conclusions}

Although we recognise that immunisation is indispensable for the global elimination of various diseases, this large-scale birth cohort study demonstrated that the prevalence of asthma, wheeze and eczema in children at 12 months of age was associated with the administration of a larger number of types of inactivated vaccines at the initial immunisation before 6 months of age. Despite this association, we strongly support the global vaccination strategy and do not recommend that immunisation be halted. The results of this research support the reconsideration of better vaccination development in the future. Nonetheless, we highly encourage immunisation and support its current global strategy. The present results should be cautiously interpreted, but they suggest that the development of better vaccines and/or adjuvants could be beneficial.

\section{Supplementary information}

Supplementary information accompanies this paper at https://doi.org/10. 1186/s12199-020-00864-7.

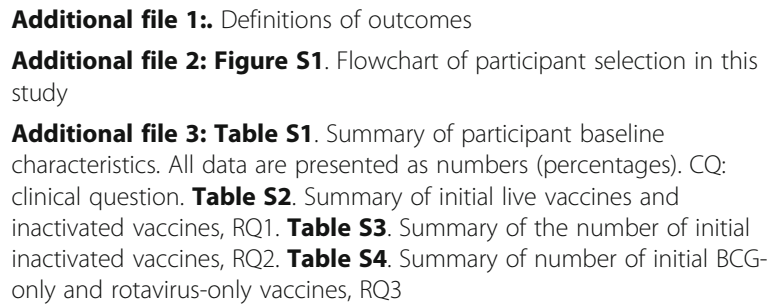

Additional file 4: Table S5. Prevalence for $R Q 1$

Additional file 5: Table S6. Prevalence for RQ2

Additional file 6: Table S7. Prevalence for $\mathrm{RQ3}$

\section{Abbreviations}

aOR: Adjusted odds ratio; BCG: Bacillus Calmette-Guérin; Cl: Confidence interval; CQ: Clinical question; Crl: Credible interval; DTaP: Diphtheria, tetanus and acellular pertussis; Hib: Haemophilus influenza type b; IL: Interleukin; JECS: Japan Environment and Children's Study; ISAAC: The International Study of Asthma and Allergies in Childhood; OR: Odds ratio; OTC: Over-thecounter; PCV: Pneumococcal conjugate vaccine; Pr: Probability; RCT: Randomised controlled trial; Th: T helper; UNICEF: United Nations Children's Fund; WHO: World Health Organization

\section{Acknowledgements}

We would like to thank the children and their families for participating in the JECS. This study was funded and supported by the Ministry of the Environment, Japan. The findings and conclusions of this article are solely the responsibility of the authors and do not represent the official views of the above government agency. Members of the JECS (principal investigator, Kamijima Michihiro) as of 2019 include: Shin Yamazaki (National Institute for Environmental Studies, Tsukuba, Japan), Yukihiro Ohya (National Center for Child Health and Development, Tokyo, Japan), Reiko Kishi (Hokkaido University, Sapporo, Japan), Nobuo Yaegashi (Tohoku University, Sendai, Japan), Koichi Hashimoto (Fukushima Medical University, Fukushima, Japan), Chisato Mori (Chiba University, Chiba, Japan), Shuichi Ito (Yokohama City 
University, Yokohama, Japan), Zentaro Yamagata (University of Yamanashi, Chuo, Japan), Hidekuni Inadera (University of Toyama, Toyama, Japan), Michihiro Kamijima (Nagoya City University, Nagoya, Japan), Takeo Nakayama (Kyoto University, Kyoto, Japan), Hiroyasu Iso (Osaka University, Suita, Japan), Masayuki Shima (Hyogo College of Medicine, Nishinomiya, Japan), Youichi Kurozawa (Tottori University, Yonago, Japan), Narufumi Suganuma (Kochi University, Nankoku, Japan), Koichi Kusuhara (University of Occupational and Environmental Health, Kitakyushu, Japan) and Takahiko Katoh (Kumamoto University, Kumamoto, Japan). Special thanks to Mr. Lawrence W. Stiver (Quality Translation Co., Ltd.; Tokyo, Japan; qualityt@gol.com) for proofreading the manuscript. We also thank Katie Oakley, PhD, from Edanz Group (https://en-author-services.edanzgroup.com/) for editing a draft of this manuscript.

\section{Authors' contributions}

$\mathrm{KYH}, \mathrm{MSA}$, MiS and YO contributed to the study design. KYH, LY and PK contributed to the statistical plan. PK analysed the data and prepared the study results. KYH, MSA, MiS, LY, HM, HS, MN, MK, KI, KM, HS and YO contributed to the interpretation of the findings. $\mathrm{KYH}$ led the drafting of the manuscript, and all co-authors contributed to revising of the manuscript and approved the final version.

\section{Funding}

The Japan Environment and Children's Study was funded by the Ministry of the Environment, Japan. The findings and conclusions of this study are the sole responsibility of the authors and do not represent the official views of the Japanese Government

\section{Availability of data and materials}

The data used to derive our conclusions are unsuitable for public deposition due to ethical restrictions and specific legal framework in Japan. It is prohibited by the Act on the Protection of Personal Information (Act No. 57 of 30 May 2003, amended on 9 September 2015) to publicly deposit data containing personal information. The Ethical Guidelines for Epidemiological Research enforced by the Japan Ministry of Education, Culture, Sports, Science and Technology and the Ministry of Health, Labour and Welfare also restrict the open sharing of the epidemiologic data. All inquiries about access to data should be sent to jecs-en@nies.go.jp. The person responsible for handling inquiries sent to this e-mail address is Dr Shoji F. Nakayama, JECS Programme Office, National Institute for Environmental Studies.

\section{Ethics approval and consent to participate}

The study protocol was approved by the Ministry of the Environment's Institutional Review Board on Epidemiological Studies as well as the ethics committees of all participating institutions. All the participants provided written informed consent.

\section{Consent for publication}

\section{Not applicable.}

\section{Competing interests}

The authors declare that they have no competing interests.

\section{Author details}

'Allergy Center, National Center for Child Health and Development, Tokyo, Japan. ${ }^{2}$ Medical Support Center for the Japan Environment and Children's Study, National Research Institute for Child Health and Development, Tokyo, Japan. ${ }^{3}$ Division of Biostatistics, Department of Data Management, Center for Clinical Research and Development, National Center for Child Health and Development, Tokyo, Japan. ${ }^{4}$ Department of Allergy and Clinical Immunology, National Research Institute for Child Health and Development, Tokyo, Japan.

Received: 5 February 2020 Accepted: 18 June 2020

Published online: 07 July 2020

\section{References}

1. Marciani DJ. Vaccine adjuvants: role and mechanisms of action in vaccine immunogenicity. Drug Discov Today. 2003;8:934-43.
2. Marrack P, McKee AS, Munks MW. Towards an understanding of the adjuvant action of aluminium. Nat Rev Immunol. 2009;9:287 https://doi.org/ 10.1038/nri2510

3. Centers for Disease Control and Prevention. Adjuvants help vaccines work better. 2018. https://www.cdc.gov/vaccinesafety/concerns/adjuvants.html. Accessed 22 July 2019

4. Yamanishi R, Yusa I, Bando N, TERAO J. Adjuvant activity of alum in inducing antigen specific lgE antibodies in BALB/C mice: a reevaluation. Biosci Biotechnol Biochem. 2003;67:166-9.

5. Kiraly N, Koplin JJ, Crawford NW, Bannister S, Flanagan KL, Holt PG, et al. Timing of routine infant vaccinations and risk of food allergy and eczema at one year of age. Allergy. 2016;71:541-9 https://doi.org/10.1111/all.12830.

6. Schlaud M, Schmitz R, Poethko-Muller $C$, Kuhnert R. Vaccinations in the first year of life and risk of atopic disease-results from the KiGGS study. Vaccine. 2017:35:5156-62 https://doi.org/10.1016/j.vaccine.2017.07.111.

7. Spycher BD, Silverman M, Egger M, Zwahlen M, Kuehni CE. Routine vaccination against pertussis and the risk of childhood asthma: a population-based cohort study. Pediatrics. 2009;123:944-50 https://doi.org/ 10.1542/peds.2008-0115.

8. Vogt H, Bråbäck L, Kling A-M, Grünewald M, Nilsson L. Pertussis immunization in infancy and adolescent asthma medication. Pediatrics. 2014;134:721.

9. Kleinnijenhuis J, Quintin J, Preijers F, Benn CS, Joosten LA, Jacobs C, et al. Long-lasting effects of BCG vaccination on both heterologous Th1/Th17 responses and innate trained immunity. J Innate Immun. 2014;6:152-8 https://doi.org/10.1159/000355628.

10. Linehan MF, Nurmatov U, Frank TL, Niven RM, Baxter DN, Sheikh A. Does $B C G$ vaccination protect against childhood asthma? Final results from the Manchester Community Asthma Study retrospective cohort study and updated systematic review and meta-analysis. J Allergy Clin Immunol. 2014; 133:688-95 e14 https://doi.org/10.1016/j.jaci.2013.08.007.

11. Yamamoto-Hanada K, Yang L, Narita M, Saito H, Ohya Y. Influence of antibiotic use in early childhood on asthma and allergic diseases at age 5 . Ann Allergy Asthma Immunol. 2017;119:54-8 https://doi.org/10.1016/j.anai. 2017.05.013

12. Yamamoto-Hanada K, Yang L, Ishitsuka K, Ayabe T, Mezawa H, Konishi M, et al. Allergic profiles of mothers and fathers in the Japan Environment and Children's Study (JECS): a nationwide birth cohort study. World Allergy Organ J. 2017:10:24 https://doi.org/10.1186/s40413-017-0157-0.

13. Kawamoto T, Nitta H, Murata K, Toda E, Tsukamoto N, Hasegawa M, et al. Rationale and study design of the Japan environment and children's study (JECS). BMC Public Health. 2014;14:25 https://doi.org/10.1186/1471-2458-14-25.

14. Michikawa T, Nitta H, Nakayama SF, Ono M, Yonemoto J, Tamura K, et al. The Japan Environment and Children's Study (JECS): a preliminary report on selected characteristics of approximately 10000 pregnant women recruited during the first year of the study. J Epidemiol. 2015;25:452-8 https://doi.org/ 10.2188/jea.JE20140186.

15. Yang L, Yamamoto-Hanada K, Ishitsuka K, Ayabe T, Mezawa H, Konishi M, et al. Medical and surgical complications in pregnancy and obstetric labour complications in the Japan Environment and Children's Study (JECS) cohort: a birth cohort study. J Obstet Gynaecol. 2019; https://doi.org/10.1080/ 01443615.2019.1673709.1-7, https://doi.org/10.1080/01443615.2019.1673709.

16. Asher M, Keil U, Anderson H, Beasley R, Crane J, Martinez F, et al. International study of asthma and allergies in childhood (ISAAC): rationale and methods. Eur Respir J. 1995;8:483-91.

17. The National Institute of Infectious Diseases (NIID) Japan. Immunization Schedule, Japan 2011 (for those under 20 years of age). 2011. https://idsc. niid.go.jp/vaccine/dschedule/2011/Imm11EN.pdf. Accessed 22 July 2019.

18. The National Institute of Infectious Diseases (NIID) Japan. Routine and voluntary vaccinations in Japan (Under 20 years old) (Effective Since November 1, 2012). 2012. https://www.niid.go.jp/niid/images/vaccine/ schedule/2012/ImmEN121101.pdf. Accessed 22 July 2019.

19. Groothuis-Oudshoorn K, Van Buuren S. Mice: multivariate imputation by chained equations in R. J Stat Softw. 2011:45:1-67.

20. Team SD: RStan: the $R$ interface to Stan. $R$ package version 2.17.3. 2018. URL http://mc-stan.org/. Accessed July 222019.

21. Team RC. R: A language and environment for statistical computing. Vienna: R Foundation for Statistical Computing; 2018. https://www.R-project.org/. Accessed 22 July 2019.

22. McDonald KL, Huq SI, Lix LM, Becker AB, Kozyrskyj AL. Delay in diphtheria, pertussis, tetanus vaccination is associate with a reduced risk of childhood asthma. J Allergy Clin Immunol. 2008;121(3):626-31. 
23. Simon AK, Hollander GA, McMichael A. Evolution of the immune system in humans from infancy to old age. Proc Biol Sci. 2015;282:20143085 https:// doi.org/10.1098/rspb.2014.3085.

24. Yang L, Narita M, Yamamoto-Hanada K, Sakamoto N, Saito H, Ohya Y. Phenotypes of childhood wheeze in Japanese children: A group-based trajectory analysis. Pediatr Allergy Immunol. 2018;29(6):606-11 https://doi. org/10.1111/pai.12917

25. Kashiwagi Y, Miyata A, Kumagai T, Maehara K, Suzuki E, Nagai T, et al. Production of inflammatory cytokines in response to diphtheria-pertussistetanus (DPT), haemophilus influenzaetype b (Hib), and 7-valent pneumococcal (PCV7) vaccines. Hum Vaccin Immunotherapeutics. 2014;10: 677-85 https://doi.org/10.4161/hv.27264.

26. von Hertzen L, Klaukka T, Mattila H, Haahtela T. Mycobacterium tuberculosis infection and the subsequent development of asthma and allergic conditions. J Allergy Clin Immunol. 1999:104:1211-4.

27. Shirakawa T, Enomoto T, Shimazu S-I, Hopkin JM. The inverse association between tuberculin responses and atopic disorder. Science. 1997;275:77-9.

28. HogenEsch H. Mechanism of immunopotentiation and safety of aluminum adjuvants. Front Immunol. 2013;3:406.

29. Onishi M, Ozasa K, Kobiyama K, Ohata K, Kitano M, Taniguchi K, et al. Hydroxypropyl-beta-cyclodextrin spikes local inflammation that induces Th2 cell and T follicular helper cell responses to the coadministered antigen. J Immunol. 2015;194:2673-82 https://doi.org/10.4049/jimmunol.1402027.

30. Sonnenschein-van der Voort AM, Arends LR, de Jongste JC, Annesi-Maesano I, Arshad SH, Barros H, et al. Preterm birth, infant weight gain, and childhood asthma risk: a meta-analysis of 147,000 European children. J Allergy Clin Immunol. 2014;133:1317-29 https://doi.org/10.1016/j.jaci.2013. 12.1082

\section{Publisher's Note}

Springer Nature remains neutral with regard to jurisdictional claims in published maps and institutional affiliations.

Ready to submit your research? Choose BMC and benefit from:

- fast, convenient online submission

- thorough peer review by experienced researchers in your field

- rapid publication on acceptance

- support for research data, including large and complex data types

- gold Open Access which fosters wider collaboration and increased citations

- maximum visibility for your research: over $100 \mathrm{M}$ website views per year

At $\mathrm{BMC}$, research is always in progress.

Learn more biomedcentral.com/submissions 